\title{
STUDENTS' ABILITY TO MAKE SENSE OF ALGEBRAIC EXPRESSIONS AND THEIR VERBAL EQUIVALENTS ${ }^{i}$
}

\author{
Emrullah Erdem ${ }^{1 i i}$, \\ Şeyda Zengin ${ }^{2}$, \\ Hayrullah Erdem ${ }^{3}$ \\ ${ }^{1}$ Assoc. Prof. Dr., \\ Adiyaman University, \\ Faculty of Education, \\ Department of Mathematics Education, 02040, \\ Adıyaman, Turkey \\ ${ }^{2}$ Teacher, Ministry of National Education, \\ Bingöl, Turkey \\ ${ }^{3}$ Teacher, Ministry of National Education, \\ Adiyaman, Turkey
}

\begin{abstract}
:
This study aims to reveal the 6th-grade students' ability to write and understand the verbal expressions (VE) of algebraic expressions (AE) and AE of VE. 238 sixth graders studying in five middle schools in two provinces of Turkey participated in the study. Algebraic Expressions Test (AET), consisting of 24 items, was developed and used as a data collection tool. In the research, quantitative data were analyzed with a t-test and qualitative data were analyzed with content analysis technique. Evidence was found that the performance of the participants was above the average $(\bar{x}=2,05)$. There was no significant difference between the achievements of male students and female students. On the other hand, it was determined that the students ignored the parenthesis, did not pay attention to the priority of operation and the priority of the fraction line, couldn't comprehend equality, generally wrote the same type of VE, and could not interpret AE.
\end{abstract}

Keywords: Algebra, algebraic expressions, verbal expressions, 6th-grade students

\section{Introduction}

While some of the mathematics subjects are basic and easy to learn, some of them are abstract and difficult to learn and teach. Do students have difficulties in writing and making sense of Algebraic Expressions (AE) that form the basis of algebra? The present

\footnotetext{
'A part of this study was presented at the 12th National Science and Mathematics Education Congress in Turkey.

ii Correspondence: email eerdem@outlook.com, zngseyda@gmail.com, hayrullaherdem@hotmail.com
} 
study is basically about this problem. It is not difficult to guess that many people will answer 'Yes' to this question. The reason for this inference can be given that "Algebra" is one of the abstract subjects of mathematics (Kieran, 1992). Algebra is a unique language of mathematics that expresses itself with symbols, tables, words, and graphics (Stacey \& MacGregor, 2000). Algebra fully reveals the slogan "mathematics is the science of abstractions" and can pave the way for moving away from mathematics when it is not learned meaningfully. For example, a student who can easily perform the $3+4=7$ arithmetic operation may have difficulty in performing an algebraic operation in the form of $3+x=7$. This transition from arithmetic to algebra is an extremely important process that develops the abstract thinking skill required for mathematics. Kieran (2004) pointed out that students generally focus on calculating rather than comprehending the relational aspects of operations, and underlined that it is necessary to pay attention to the following adjustments for successful algebraic thinking (p. 140-141):

1) A focus on relations and not merely on the calculation of a numerical answer;

2) A focus on operations as well as their inverses, and on the related idea of doing / undoing;

3) A focus on both representing and solving a problem rather than on merely solving it;

4) A focus on both numbers and letters, rather than on numbers alone. This includes:

a. working with letters that may at times be unknowns, variables, or parameters;

b. accepting unclosed literal expressions as responses;

c. comparing expressions for equivalence based on properties rather than on numerical evaluation;

5) A refocusing of the meaning of the equal sign.

\section{Literature Framework of the Difficulties in Algebra}

It is underlined by NCTM (2000) that algebra has a more abstract structure than arithmetic and therefore students have various difficulties while learning it. Students may experience various difficulties while learning algebra and may fall into misconceptions. For example, Booth (1988) grouped the difficulties and misconceptions that students experienced while using and interpreting AE under four headings. (1) Focusing on the answer: the students cannot make sense of the algebraic notation process, they are looking for a numerical solution and cannot even write the correct result as the correct answer, (2) algebraic notation and the use of rules: the students try to write the result of the expression $2 a+5 b$ as $7 a b$. (3) meaning of letters and variables: students mostly think of 3 apples in the AE 3a, not "3 times the number of apples", (4) types of methods and relations used in arithmetic: when students' arithmetic knowledge is incomplete and incorrect, their learning of algebra is also negatively affected. In addition, students ignore the use of parentheses and do not pay attention to the order of operations. In another study (Perso, 1992), students' misconceptions about AE were combined into three categories: (1) not understanding the role of letters in algebra, (2) not being able to use variables, and (3) not using algebraic rules while solving equations. 
Studies (eg, Kieran, 1992; Nathan \& Koedinger, 2000) have revealed different reasons for students' difficulties in algebra. For example, teacher-induced differences in the teaching of algebra can affect students' learning and mental activities. For this reason, it is a necessity to learn the first concepts well in the transition to algebra, to know the possible misconceptions of the students, and to carry out activities to remedy them. Similarly, it is emphasized that teachers should teach algebra to their students in a way that maximizes their comprehension and retention levels (Leitze \& Kitt, 2000). In another study, it was found that the difficulties experienced by students in algebra stem from the student's inability to understand the concept of variable sufficiently, not knowing the difference between "unknown" and "variable" concepts, lack of knowledge in arithmetic, and lack of time given for teaching algebra (Şimşek \& Soylu, 2018). It is important for teachers to know these difficulties and to teach in this direction in terms of effective algebra learning.

\subsection{Literature Review on Teaching Algebra}

In this section, the researches on algebra are presented in detail from the past to the present, what kind of research has been done in the literature, and what kind of results have been reached. Thus, both researchers, teachers, and teacher candidates will be guided on how algebra should be taught and what kind of research is needed. For example, in the study conducted by Sharp (1995), various materials and activities were used to teach addition, subtraction, multiplication, and division operations in AE to the students in the experimental group, while the students in the control group were taught with traditional methods. The results of the research showed that the use of various concrete materials and activities in the teaching of AE both increased success and allowed students to learn conceptually by seeing different representations of the algebra concepts. Akkan et al. (2012) examined and compared the problem-solving processes of 5th-8th grade students during their transition from arithmetic to algebra. It was found out that as the age increased, the transition from arithmetic solutions to algebraic solutions did not develop at the desired level, and students from different education levels still used arithmetic solutions instead of algebraic solutions. In their study, Bağdat and AnapaSaban (2014) investigated the algebraic thinking skills of 8th-grade students, which are listed as formulating generalizations, using symbols and algebraic relations, and making use of multiple representations, using SOLO Taxonomy. They carried out the process with the participation of 15 8th-grade students. Eight problems were used and clinical interviews were conducted with the students on these problems. As a result of the research, it was revealed that the students mostly had difficulties in using symbols and AE. Susac et al. (2014) conducted a study with 7th and 8th-grade middle school students and 9th and 10th-grade high school students to examine the development of abstract mathematical reasoning. To do this, they used equations containing simple AE. In the study, it was observed that high school students reasoned more abstractly (using letters instead of numbers, using equation solving strategies, etc.) in their solutions compared to middle school students. Based on the results of the research, it was emphasized that better planning is needed for the timing of the students' transition from arithmetic 
reasoning to abstract algebraic reasoning. In this context, to make the transition from arithmetic to algebra successful, it is necessary to focus on numbers and letters together rather than just numbers (Kieran, 2004). Şimşek and Soylu (2018) examined the difficulties experienced by 7th-grade students in AE and the reasons for these difficulties. The algebra knowledge test created for this purpose was applied to the 7th-grade students and the reasons for the difficulties experienced were determined through semistructured interviews. As a result of the research, it was revealed that the students had difficulties such as not noticing the variable, converting the AE into an equation, using " $x$ " mostly as a variable, and incorrectly constructing the equation suitable for the problem. In the interviews, the reasons for them were stated as the student's inability to attribute a meaning to the variable in the operation, not being able to distinguish the concepts of unknown and variable, identifying the variable with the expression " $x$ ", lack of knowledge in arithmetic operations, and insufficient time allocated to algebra. Hemmi et al. (2021) conducted a study to compare the algebra teaching curricula of three different countries. It was thought that it would be more appropriate to give the similar beneficial aspects of the three curriculum contexts in this section rather than the advantages and/or deficiencies of the countries in algebra teaching. In the study, the following common suggestions have been identified in algebra teaching in three countries: (1) early introduction of the relationship between the concepts of "unknown number" and "variable", (2) the use of the inverse property of arithmetic operations is very important both in exams and while solving equations, (3) it is extremely necessary to give the relations between arithmetic operations and arithmetic rules starting from the 1st grade for generalized arithmetic in the future, (4) proportional reasoning and percentage concepts are also necessary for successful algebra teaching.

When the studies are examined, it is seen that the studies generally focus on detecting errors and misconceptions about algebra. More research has been conducted on students in the 7th and 8th grades of middle school. Various suggestions were presented by identifying the misconceptions experienced by the students in the research. In addition, teachers' views on teaching algebra and the difficulties experienced by students while learning are also among the topics examined. The current research, unlike these studies, is important in terms of determining the ability of 6th-grade students to write and make sense of AE they encounter for the first time and to make a different contribution to the literature in this respect.

\subsection{The Role of Reading Comprehension (RC)}

To write VE and their AE, first of all, it is necessary to understand that expression well. To do this, besides sufficient mathematical reasoning skill, reading comprehension skill is needed. In this context, it would be useful to briefly mention the researches on RC skill. $\mathrm{RC}$ is a basic skill required for everyone as it enables better thinking (Danaei et al., 2020). $\mathrm{RC}$ is defined as an active process that associates prior knowledge and experience with new knowledge to construct the meaning in the text read (Kao et al., 2016). Mathematical operations and RC require similar mental processes (Ural \& Ülper, 2013). Similarly, Knopik and DeFries (1999) stated that reading and mathematics generally affect each 
other and their deficiencies occur together. In mathematics, students' ability to re-express an expression with their sentences is one of the basic skills that should be acquired. Understanding a mathematical expression is a prerequisite for making that expression and the processes in which this expression is used productive (Erdem, 2016). When someone's RC skill is insufficient, related mathematical expressions can be written and interpreted incorrectly. For example, when writing the AE of VE such as "Two less than three times one less of the walnuts in the box", it is important to understand the words correctly with each other. Otherwise, the student may not fully understand this VE and write the wrong AE as " $[(3 x-1)]-2$ " or " $[(x-1)-2] .3$ ".

The existence of the relationship between mathematical reasoning and RC shows itself in the first years of someone's education life. Giving the foundations of reading and mathematics together from preschool can be shown as an example of this (Erdem, 2016). In the literature, it is suggested that early literacy skill is not only a predictor of future reading skill but also a predictor of future mathematics achievement (Birgisdottir et al., 2020). Vilenius-Tuohimaa et al. (2008) found a strong relationship between these two skills as a result of their study to reveal the relationship between mathematical problemsolving skill and RC. Similarly, in a study conducted with middle school 8th-grade students, it was determined that there was a positive and significant relationship between mathematical reasoning skill and RC (Erdem, 2016). Passolunghi and Pazzaglia (2005) conducted a study to examine and compare the skills of high and low-level problem solvers. Research results have shown that to be a good problem solver, it is necessary to be at a good level in RC. Based on these studies, it can be said that RC is necessary for writing the AE of VE.

\section{The Current Study}

Learning the concepts that form the basis of algebra well is effective in knowing and remedying possible misconceptions about algebra. Being able to determine the AE of VE is the basis for solving problems and establishing equations correctly. As it is known, the most important step in problem-solving is understanding the problem. In problems whose solution requires using variables, it is very important to be able to write and interpret $\mathrm{AE}$ to understand the problem. Even if the problem is understood, it may be difficult to solve if appropriate AE cannot be written or AE cannot be understood correctly. This difficulty is likely to increase as the grade level increases. In other words, students' problems in learning algebra will be reflected in their future mathematics education, and these problems will continue to increase gradually. Making sense of AE has an important role in the effective realization of high-level mathematics teaching in the future. As stated earlier, when the literature is examined, it is seen that most of the studies on algebra were conducted with 7th and 8th-grade students. There are not many studies examining the ability of 6th-grade students to make sense of AE. In this context, this study aims to determine the 6th-grade students' ability to write and make sense of the VE of AE and AE of VE. It is thought that this study will also provide resources to the 
literature to make sense of AE at the 6th-grade level. In the present study, answers to the following items were sought.

1) What is the level of 6th-grade students' ability to write and make sense of the VE of $\mathrm{AE}$ and $\mathrm{AE}$ of VE?

2) Does the performance of 6th-grade students differ by gender?

3) What kind of difficulties do 6th-grade students have in this process?

\section{Methodology}

\subsection{Research Design}

In the present study, a mixed (quantitative + qualitative) research design was used. It is quantitative research because the ability of the participants to write and make sense of AE through a scale is demonstrated by descriptive statistics. The answers given by the participants to the items in the scale are also subjected to content analysis and categories are created, so it is qualitative research. Mixed studies allow us to deal with research problems in more detail and with different aspects compared to the preference of quantitative and qualitative methods alone (Creswell, 2003). In the research, a more detailed picture of how the participants think was reached by examining the answers obtained through quantitative means, as well as qualitative ways.

\subsection{Subjects}

The research was carried out with the participation of 238 sixth-grade students from five middle schools in different socio-economic environments in two provinces of Turkey. These students were selected from a low, medium, and high achievement branch in each school. Maximum diversity sampling was used to ensure that the research results are as representative of students in this age group as possible. In this context, it was ensured that the schools were different in terms of success ( 2 low, 2 medium, 1 high) by interviewing the administrators and teachers of the schools where this study would be conducted. The success status of the selected schools was confirmed by obtaining information from the Provincial Directorates of National Education. 112 of the participants are female students and 126 of them are male students. The subject of AE is taught at the 6th and 7th-grade levels according to the Middle School Mathematics Course $(5,6,7,8)$ Curriculum (MNE, 2018). Since writing the VE of AE is mainly given at the sixth-grade level, students in this grade were included in the study. To keep their identities secret, the students were given codes such as S1, S2, S3, ...

\subsection{Data Collection}

As a data collection tool, the Algebraic Expressions Test (AET) consisting of 24 items, developed by making use of the MNE (2018) curriculum and middle school mathematics textbooks, was prepared. Table 1 shows some items regarding the learning outcomes of "A student writes the VE of $A E$ " and "A student writes the AE for which the VE is given", which are expected to be achieved by the 6th-grade students in the MNE (2018). 


\begin{tabular}{|c|c|}
\hline $\begin{array}{c}\text { Items for } \\
\text { writing the VE of AE }\end{array}$ & $\begin{array}{c}\text { Items for } \\
\text { writing AE of VE }\end{array}$ \\
\hline - $9(c-3)+5$ & $\begin{array}{l}\text { - An algebraic expression that "gives the } \\
\text { perimeter of an equilateral triangle with a } \\
\text { side of } \mathrm{k} \mathrm{cm} \text { " }\end{array}$ \\
\hline - $\frac{a}{5}+2=21$ & $\begin{array}{l}\text { - An algebraic expression that gives "two less } \\
\text { than three times one less of the walnuts in the } \\
\text { box" }\end{array}$ \\
\hline - $\frac{n-6}{4}=8$ & $\begin{array}{l}\text { - An algebraic expression that gives "Ali's } \\
\text { remaining debt, who pays } 3 \text { TL of his debt" }\end{array}$ \\
\hline - $\frac{5 M-4}{3}$ & $\begin{array}{l}\text { - If the price of a 50-kilogram bag of sugar is } x \\
\mathrm{TL} \text {, the algebraic expression that gives the } \\
\text { price of } 5 \text { kilograms of sugar }\end{array}$ \\
\hline
\end{tabular}

The average score that can be obtained from the test is at least 0 , and the maximum is 3 . To determine whether the items in the test are items for writing the VE of AE and AE of $\mathrm{VE}$, the opinions of two experienced mathematics teachers and a mathematics education specialist were taken. It was also checked whether the items were understandable and whether there were possible grammatical errors in the items by a researcher Turkishlanguage teacher. As a result of the pilot application conducted with three students with different achievements, it was decided that 40 minutes was appropriate for the test and it was seen that the students could understand the items. Item analysis was conducted to finalize the items in the test. The lowest item-total correlation coefficient was found to be 0.391. It is stated that items with an item-total correlation of .30 and higher distinguish individuals well, items between .20 and .30 can be included in the test or should be corrected if it is deemed necessary, and items with a lower than .20 should be excluded from the test (Büyüköztürk, 2011). In addition, the Cronbach Alpha coefficient of the AET was calculated as .924 . The results of this analysis show that the test can be applied reliably for students at this level.

\subsection{Data Analysis}

The answers given to the items in the AET were scored in four stages, with Completely True " 3 ", Partially True " 2 ", Incorrect " 1 ", and No Answer " 0 ". The average of the scores of all students from the test was calculated separately. The answers to all items in the test were scored independently by two mathematics education experts. The Pearson Correlation Coefficient ( $r$ ) was calculated to reveal the consistency between these two scores. The consistency between the scores of the two experts independently from each other was found as $90 \%(\mathrm{p}=.000, \mathrm{r}=.897)$. The Kolmogorov-Smirnov test used to determine normality showed that the data were not normally distributed $(p=.029)$. For this reason, the Mann-Whitney $U$ test was used to reveal how achievement varies by gender. In the analysis of qualitative data, a content analysis technique was used and six categories agreed upon by the two researchers were reached. To ensure coding reliability, Miles and Huberman's (1994) reliability formula (reliability=consensus/(consensus+dissensus) was used. For this study, coding reliability was calculated as 0.87 . On the other hand, some 
student answers were directly quoted to increase the reliability of the research. In addition, after the real application was carried out and all student papers were scored, interviews were conducted with students whose answers were not well understood, and they were allowed to explain their answers in more detail. In this context, sample interviews of three students were directly conveyed as Yin (2011) suggested in qualitative evaluations.

\section{Findings}

In this section, it is given whether the performance of the participants varies according to gender. In addition, the writing and interpretation situations of the students were presented as categories. The results of the participants' success by gender are given in Table 2.

Table 2: Statistical results of success by gender

\begin{tabular}{|l|l|c|c|c|c|c|}
\hline \multicolumn{2}{|c|}{ Gender } & $\mathbf{N}$ & Mean Rank & Sum of Ranks & Sig. (2-tailed) & Z \\
\hline \multirow{3}{*}{ Success } & Female & 112 & 125,55 & 14062,00 & & \multirow{2}{*}{, 201} \\
\cline { 2 - 5 } & Male & 126 & 114,12 & 14379,00 & $-1,279$ \\
\hline & Total & 238 & & & & \\
\hline
\end{tabular}

As can be seen from Table 2, there was no significant difference between the success of male and female students' performance $(z=-1.279, p=.201)$. Considering the mean rank, it can be seen that gender does not have a significant effect. In other words, it was determined that the gender factor did not have a significant effect on writing and making sense of the VE of AE and AE of VE.

Table 3: Success levels and averages of the participants

\begin{tabular}{|l|c|c|c|}
\hline Test Average / Level & $\mathbf{N}$ & Percent (\%) & Average \\
\hline $0,00-0,99 /$ Low & 7 & 3 & \multirow{2}{*}{2,05} \\
\hline $1,00-1,99$ / Medium & 104 & 44 & \\
\hline $2,00-3,00 /$ High & 127 & 53 & \\
\hline Total & $\mathbf{2 3 8}$ & $\mathbf{1 0 0}$ & \\
\hline
\end{tabular}

As can be seen in Table 3, it was revealed that the average success of all participants was 2.05. On the other hand, it was determined that most of the participants had a high (53\%) and medium (44\%) level of success.

Table 4: Frequency and percentage of participant responses by category

\begin{tabular}{|l|c|c|}
\hline Category & $\mathbf{N}$ & Percent (\%) \\
\hline Ignoring the existence of parentheses & 109 & 46 \\
\hline Ignoring the priority of operation & 102 & 43 \\
\hline Ignoring the priority of the fraction line & 76 & 32 \\
\hline Inability to understand the concept of equality & 62 & 26 \\
\hline Writing the same type of verbal expressions & 119 & 50 \\
\hline Inability to interpret the algebraic expressions & 83 & 35 \\
\hline
\end{tabular}


As can be seen from Table 4, it was found that half of the participants (50\%) used the same type of VE. It was seen that $46 \%$ of the participants ignored the existence of parentheses. It was determined that $43 \%$ of the participants did not consider the priority of operation. On the other hand, it was seen that the participants had the least difficulty in making sense of equality $(26 \%)$. The statements of some students regarding each category are given below.

\section{a. Ignoring the Existence of Parentheses}

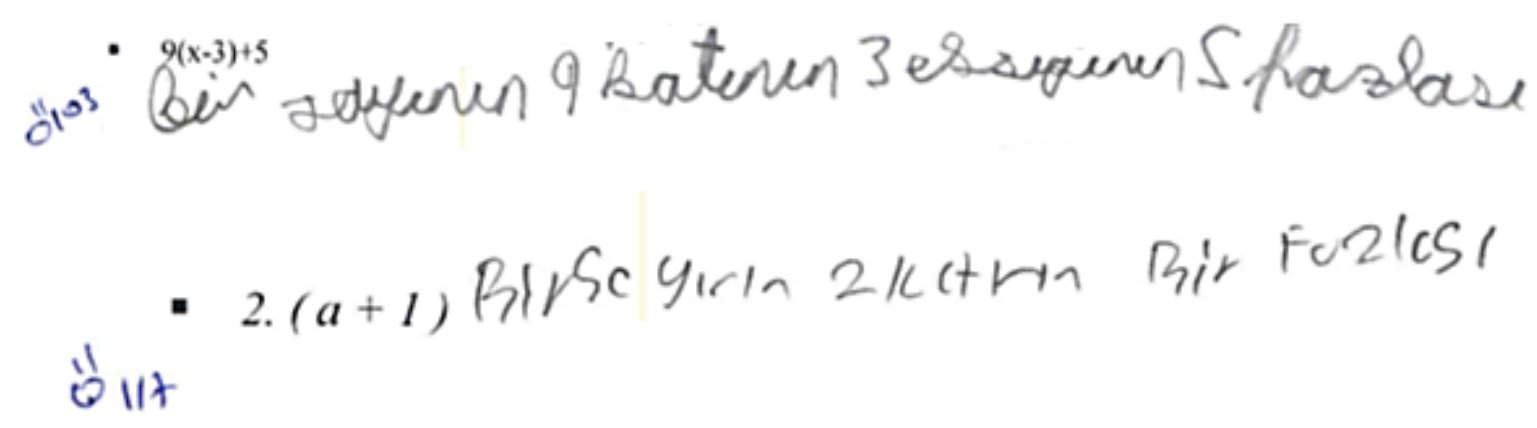

When the answers of these students were examined, it was seen that they ignored the parenthesis in writing VE of AE. S103 wrote the VE " 5 more than 3 less than 9 times a number" for the AE 9(x-3)+5. S117, on the other hand, gave the VE "1 more than twice the amount of something" as an answer for the AE 2(a+1). Both P103 and P117 could not fully grasp that parentheses are symbols that need attention while writing AE. In other words, it can be said that the students do not sufficiently comprehend that the parentheses give priority and have importance in the differentiation of the expression.

\section{b. Ignoring the Priority of Operation}

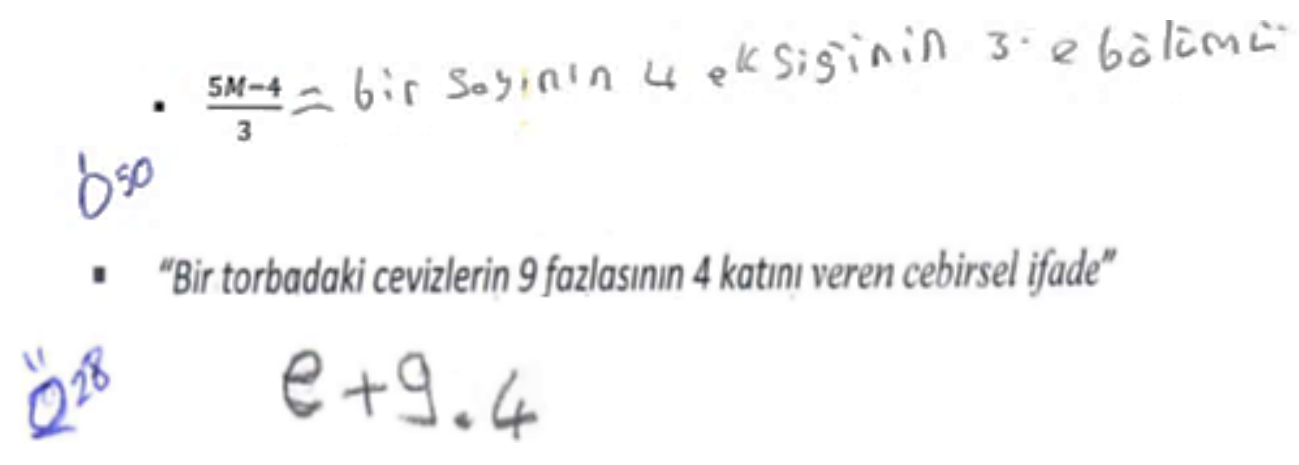

S50 wrote the VE " 4 minus of a number divided by 3" for the AE $\frac{5 M-4}{3}$. S28, on the other hand, gave the $\mathrm{AE}$ e+9.4 as an answer for the VE "AE that gives 4 times more than 9 walnuts in a box". When the answer of S50 is examined, it is understood that he did not pay attention to the priority of operation and that he did not know that the number 5 must be expressed first because of the priority of operation in the case of multiplication. When the answer of S28 is examined, it is seen that he wrote the AE incorrectly because he did not pay attention to the priority of operation. In this case, it can be said that the 
student ignores the priority of operation while reading the VE directly and writing an AE.

\section{c. Ignoring the Priority of the Fraction Line}

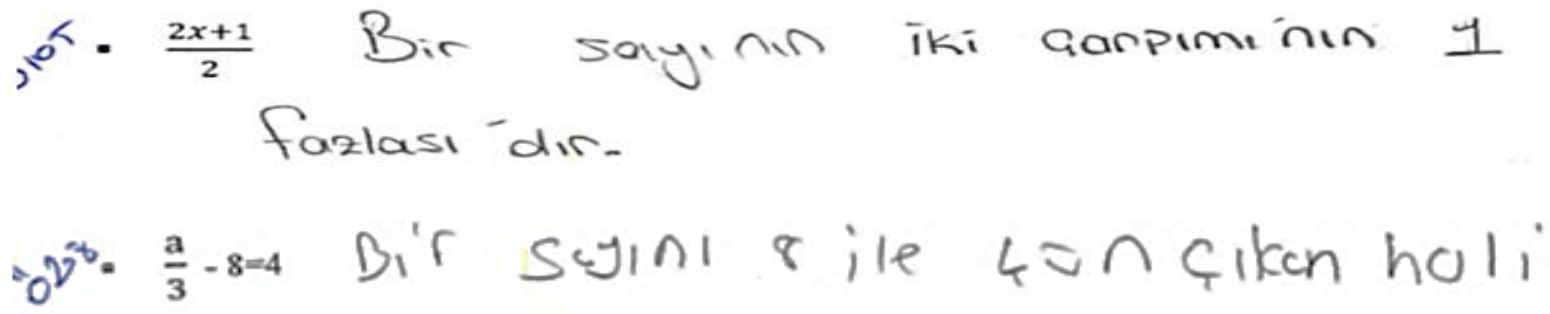

S105 wrote the VE "1 more than multiplying a number by 2 " for the AE $\frac{2 x+1}{2}$. S208, on the other hand, gave the VE " 8 and 4 subtracted from a number" as an answer for the AE $\frac{a}{3}$ $-8=4$. When S105's writing a VE for a given AE is examined, it can be said that the student did not pay attention to the fraction line and therefore wrote the VE incorrectly. The student, who paid attention to the concept of the unknown and its coefficient while writing, was able to form the expression up to a certain point of the VE but did not pay attention to the fraction line. When the answer given by S208 was examined, the student did not pay attention to the priority of the fraction line and wrote a wrong AE that was not well understood. It can be said that the student does not know that the fraction line means division and does not sufficiently comprehend that it should be expressed primarily.

\section{d. Inability to Understand the Concept of Equality}

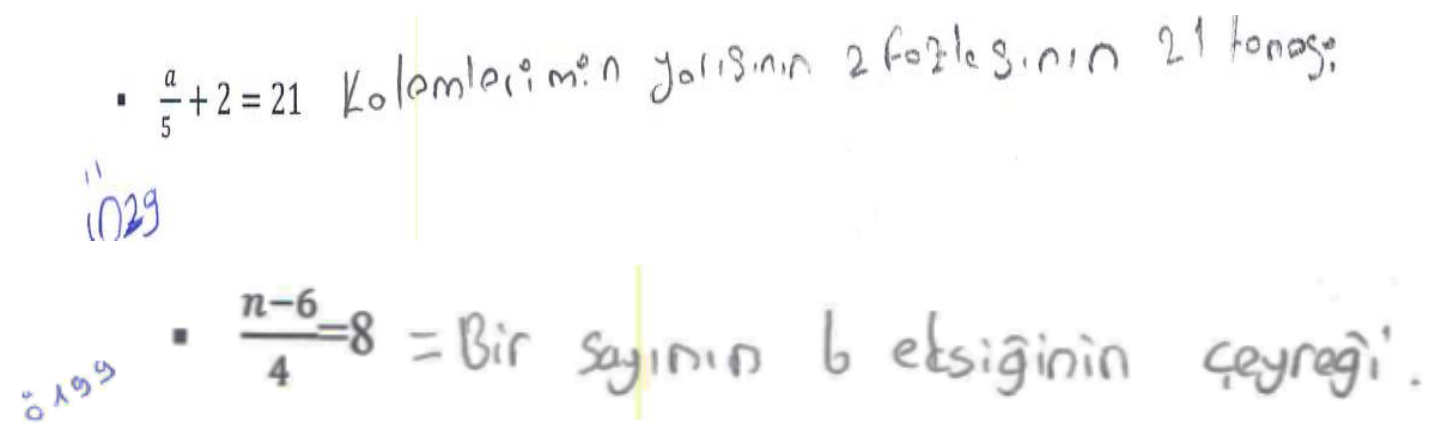

S29 wrote the VE "21 of 2 more than half of my pencils" for the $\mathrm{AE} \frac{a}{5}+2=21$. For the $\mathrm{AE} \frac{n-6}{4}=8$, S199 gave the VE "Quarter of a number minus 6" as an answer. When S29's answer is examined, it can be said that the student cannot make sense of the concept of equality. The student used the expression "number of pencils" instead of the variable "a", but ignored the concept of equality and expressed it as " 21 ". When the answer of S199 is examined, it can be said that she never used the expression "equals" and could not 
interpret that the given $\mathrm{AE}$ could be equal to a numerical value. This student read the $\mathrm{AE}$ correctly, but it was seen that she did not equate this expression to a number.

\section{e. Writing the Same Type of Verbal Expressions}

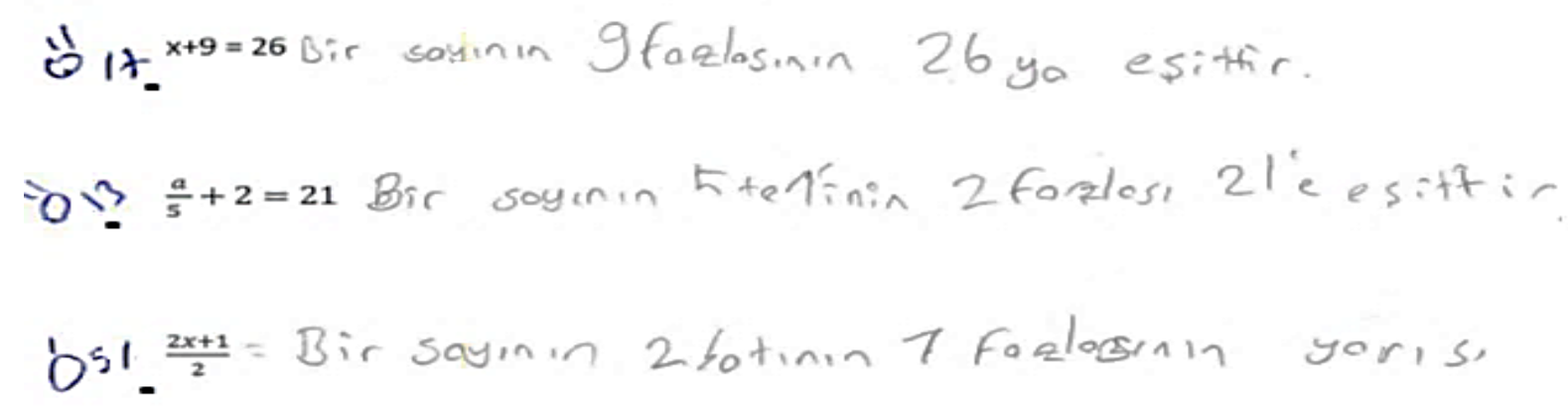

S17 wrote the VE "9 more of a number equals 26 " for the AE $\mathrm{x}+9=26 . \mathrm{S} 13$, on the other hand, gave the VE "2 more than one-fifth of a number equals 21 " as an answer for the AE $\frac{a}{5}$ $+2=21$. S51 wrote the VE "Half of 1 more than 2 times a number" for the AE $\frac{2 x+1}{2}$. As seen in these examples, it was determined that most of the students used the same type of VE while writing the VE whose AE was given. S17, S13, and S51 also wrote correct VE to AE. These students generally preferred to write VE in the form of "... a number ...".

\section{f. Inability to Interpret the Algebraic Expressions}

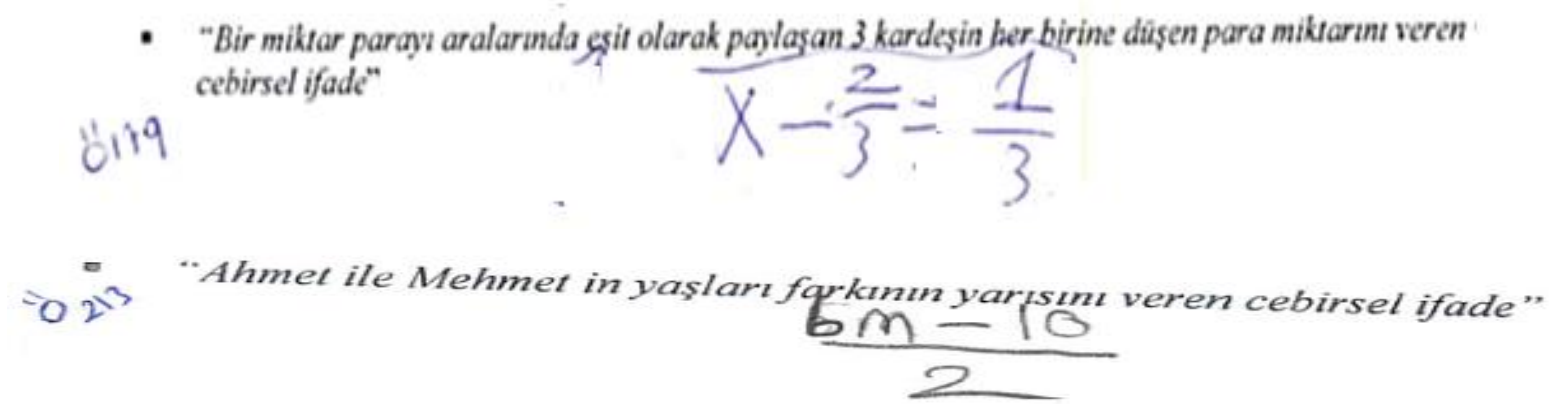

S179 wrote the AE $x-\frac{2}{3}=\frac{1}{3}$ for the VE "AE giving the amount of money falling to each of the 3 siblings who share some money equally among them". S213, on the other hand, gave the $\mathrm{AE} \frac{6 m-10}{2}$ as an answer for the VE "AE giving half the age difference of Ahmet and Mehmet". When the answer of S179 was examined, the student was able to understand that the sharing situation was division, but since he could not interpret the statement, it was seen that he wrote a wrong statement by making a few statements. When the answer of S213 is examined, it is understood that the student interpreted the number 6 as a coefficient and the number 10 as a difference, thinking that there should be numerical data in the AE. From the answer she gave, it can be said that the student could not interpret the AE correctly. The student also used constant numbers such as 6 and 10, even if incorrectly, in a situation where it was necessary to use variables. 
The interviews with students whose test successes were different were reflected and their answers to the items in the AET were detailed. Three of the interviews with students with low, medium, and high test averages are given below.

The interview with S42, whose test average was 0.83 (low level):

Researcher: Can you read the item "Algebraic expression that gives Ali's remaining debt, who pays 3 TL of his debt"?

Student: Okay...

Researcher: What's your answer?

Student: My answer is 10-3x.

Researcher: How did you think of this answer?

Student: I thought in my mind that his money is 10 TL. Then I put an $x$ next to 3 because the algebraic expression was requested.

Researcher: Okay, let's look at the other item.

Researcher: What did you think about the item "an algebraic expression that gives two less than three times one less of the walnuts in the box"?

Student: I said $6 x$ to the walnuts, I wrote $6 x$ - $1 x$ because it said 1 minus. I found the result $5 x$, I found $15 x$ because it was asked for 3 times, I wrote $15 x-13$ because it said 2 minus. Aaaa, no, I should have written $13 x$ directly. I did it wrong.

Researcher: You were asked to write the verbal equivalent of the algebraic expression $3 c+5$. As an answer, you wrote 7 minus of 8 marbles. What does the " $c$ " mean here?

Student: Algebraic expression.

Researcher: How did you think while writing this verbal expression?

Student: I subtracted 7 marbles, I was going to add 3 and 7 and add 5 again.

Researcher: But there is a parenthesis, how could you sum it up?

Student: You're right, I didn't think so.

The interview with S51, whose test average was 1.50 (medium level):

Researcher: Can you review your answer to the item "The algebraic expression that gives five more than four minus half of the chickens on a farm"?

Student: Ah. I did wrong. (He wrote "t-4+5")

Researcher: Where did you go wrong?

Student: Half means dividing something in half.

Researcher: How was it supposed to be?

Student: It should have been " $t / 2-4+5$ ".

Researcher: Can you look at item 5 ?

Student: I made a mistake (He wrote "c-1x3-2"). There is a priority of operation here, how could I not think of it, I needed to use parenthesis.

Researcher: Would anything change?

Student: Yes. It varies. It should be "(c-1)x3-2".

Researcher: What did you write as a verbal response to the item "2.(a+1)"? 
Student: 1 more than the number of pears of 2 friends. (After silence and reflection.) I thought of " 2 " as a friend, I was wrong. The unknown is the " $a$ ".

The interview with S15, whose test average was 2.62 (high level):

Researcher: Can you read item 2? ("The algebraic expression that gives five more than four minus half of the chickens on a farm") Is your answer correct?

Student: It may be true. If we give a value, for example, if we say $5,5-4=1,1+5=6,6 / 2=3$. Let's give the value 5 again. 5/2=2,5,2,5-4 (confused and leaves the solution like this by trying to perceive the difference), no result.

Researcher: Why did this happen? What is the difference?

Student: Division will be done first, but when I did the operation, the same result was not obtained.

Researcher: Could the fraction line have an effect? You drew under all the fraction lines. Student: Aaaaaaah, now I get it.

Researcher: Can you give another verbal expression for the equation " $a / 5+2=21$ "?

(In the test, he answered " 2 more than $1 / 5$ of a number is 21 ".)

Student: 2 more than one-fifth of my money is 21 TL.

Researcher: Well...

As can be understood from the sample interviews with these students, it has been observed that students with low test success have lower performance in interpreting both the items in the test and what they have written as answers. Again, in the interviews with the students, the students had the opportunity to see and correct their wrong answers. Based on these interviews, it can be said that it is necessary to examine the answers of the students in-depth and share them with them.

\section{Discussion and Conclusions}

This research was carried out to reveal the 6th-grade students' ability to write and make sense of VE of AE and AE of VE. As a result of the analyzes, the following results were obtained.

First, it was determined that the average success of the participants was 2.05. Considering that the average in the test varies between 0.00 and 3.00, it can be said that the participants' performance is above the medium level. In a study, it was concluded that middle school students' success in AE was at a moderate level and the lowest success was in "Explaining the meaning of simple visual and algebraic expressions" (Birgin \& Demirören, 2020).

Second, there was no significant difference between the success of female students and male students in AE. This result shows parallelism with the results of Ma's (1995) study on the effect of gender on success in algebra.

Third, as a result of the content analysis, it was revealed that the students ignored the existence of parentheses, did not pay attention to the priority of operation, could not pay 
attention to the priority of the fraction line, could not make sense of the concept of equality, including the same type of $V E$ and could not interpret $A E$. Similar results were obtained in the studies conducted by Özdemir-Baki and Işık (2018) on AE with 6th-grade students. In the studies conducted by Erdem and Sarpkaya-Aktaş (2018) and Şimşek and Soylu (2018), it was determined that 7th-grade students had difficulties in similar situations in AE. Falkner et al. (1999) found that students had misconceptions about the sign of equality. It is thought that it would be useful to explain these results and why they occur in more detail below.

It can be said that students' ignorance of the existence of parentheses is due to their inability to adequately grasp the concept "AE" (eg, lack of knowledge of "the operations in parentheses must be done first") and problems in RC. Since parentheses represent a priority in $\mathrm{AE}$, it requires students to interpret VE well. In other words, the parentheses give an idea from which order to start when interpreting the AE. In this context, a student who cannot comprehend the meaning of consecutive VE is likely to experience this difficulty. The fact that the concepts of algebra are not learned enough also plays a role in not paying attention to the priority of operation. It is important for teachers to convey to students why the priority of operation is necessary and how its basic logic or mathematical background is. In this context, giving the knowledge that "multiplication is repeated addition" and "division is repeated subtraction" can be effective. It can be said that the concept of fraction, which students have difficulty in understanding, is effective in not paying attention to the priority of the fraction line. Students may experience deficiencies in terms of both operational knowledge and conceptual knowledge while passing from natural numbers to fractions, and they may even have various misconceptions. Fractions, which can be confused due to their different meanings, are among the subjects that many students have difficulty in learning (Gökkurt et al., 2013; Moss \& Case, 1999; Tirosh, 2000). This difficulty may cause students to avoid fractions, and therefore the fraction line while writing VE of AE.

The fact that students encounter AE for the first time may affect their inability to make sense of the concept of equality. Students encounter AE for the first time at this grade level, and establishing and interpreting the equations in which $\mathrm{AE}$ is used is the next stage (MNE, 2018). Insufficient understanding of AE may affect the learning of this next stage more negatively. Studies have shown that students generally try to reach the result in the given equality rather than understanding the meaning of the "equality" sign (Stephens et al., 2013; Voutsina, 2019). It can be said that both the language used by the teachers and the language in the textbooks are effective in the writing of the same type of $V E$. Teachers can often use expressions that start with "A number ..." when writing or saying VE of AE. It will help to overcome this difficulty if teachers give plenty of examples of different problem sentences in which AE is used. In addition, allowing students to construct different types of problem sentences and share them with their friends can be considered as another development factor. In the textbooks, the activities on this subject should be diversified and different examples from daily life should be presented. As a matter of fact, in a study (Bozkurt, 2018), it was emphasized that the activities in the textbook were not sufficient to eliminate a possible difficulty or 
misconception. It is important to provide students with experiences that will make them realize that they are also used in real-life situations so that students do not think about the VE of AE only abstractly. The study of Dikkartın-Övez and Çınar (2018), which shows that 8th-grade students cannot solve algebra problems by associating them with daily life, also points to this necessity. Similarly, it is underlined that appropriate learning tools and methods should be used (Kieran, 2004; NCTM, 2000; Taylor-Cox, 2003; Yackel, 1997) and especially real-life examples should be used (Kieran \& Yerushalmy, 2004) in teaching algebra to students at a young age. Finally, it can be said that one of the factors affecting the students' inability to interpret $A E$ is the insufficient development of the mathematical language. In other words, this difficulty experienced when writing VE of AE and transferring them to mathematics may be due to the lack of understanding of mathematical concepts. Another factor may be the inability of students to think as a whole without considering the order of priority when transferring different judgments (difference, total, half, one-fourth, etc.) in VE to the language of mathematics. Han and Ginsburg (2001) also underlined that the language of mathematics is one of the most important skills that enable establishing correct connections between different representations, symbols, and expressions and shaping mathematics learning and thinking.

On the other hand, in the interviews conducted with some students, it was seen that the students realized their mistakes when it was examined by the researchers why they gave such answers. It can be said that this result is one of the most important practical results of the current research (especially for teachers). These interviews pointed to the importance of questioning why students think the way they think in a constructive discussion environment, rather than accepting what they wrote about algebra or any other mathematics. In this context, learning environments that allow students to help each other by seeing each other's mistakes can be prepared by teachers. In addition, based on the answers given by S42, who had a low level of success, it can be said that her knowledge of arithmetic is quite insufficient, so she could not write correct expressions while passing algebra. The success of such students can be increased by eliminating their deficiencies in arithmetic and operation knowledge, especially at lower grade levels. It was observed that S51, whose success level was at a medium level, was thought to be inadequate in terms of $\mathrm{RC}$ and that he misunderstood the VE realized his mistake thanks to the effective questions asked by the researcher. This inference supports studies that reveal the role of RC on learning mathematics and mathematical reasoning (Aiken, 1971; Erdem, 2016).

\section{Concluding Remarks and Implications}

It can be said that students' lack of mathematical language development, inadequacies in $\mathrm{RC}$, and incomplete knowledge of previous basic mathematics subjects (four operations, fractions, the priority of operation, etc.) play a role in students' difficulties in writing and making sense of VE of AE and AE of VE. If these deficiencies of the students are not corrected at a young age or when the related subject is taught for the first time, it may 
cause a more complex mathematical adventure to emerge in the future. In other words, it may be difficult to make up for it in the following years, especially when students do not understand what the concepts of "unknown" or "variable" are and what they are used for in the 6th grade when they first meet the subject. As a result, students may have difficulties in understanding the subjects they will encounter in the future, such as equations, factorizing, functions, and problem-solving. The first step in solving a problem is to understand the problem, and the second step is to develop a strategy. Being able to write problem sentences suitable for $\mathrm{AE}$ or, conversely, understanding the problem sentence and creating an appropriate AE clearly shows the relationship between making sense of $\mathrm{AE}$ and solving problems. In this context, it will be a very useful process for students at this level to write abundant and different types of AE from daily life, to create corresponding VE, and to share them with their friends and teachers. The existence of complex problems, which are popular around the world, whose results cannot be reached immediately, and which require more mathematical reasoning and different thinking processes, draws attention to the importance of AE to be sufficiently understood by students. With a longitudinal study, the relationship between students' ability to make sense of AE and their problem-solving skills can be investigated. In addition, examples, exercises, and activities used in the teaching of AE in the textbooks can be diversified, allowing students to think and interpret differently. Moreover, more time should be devoted to the teaching of this subject, which is currently tried to be given in only one learning outcome in the curriculum.

Another important point that must be emphasized in this study is that it is a limitation to conduct this research without considering or measuring students' RC skills. If the student does not have this skill yet, it can be said that $\mathrm{s} /$ he may have difficulty in writing and understanding the AE of VE. On the other hand, based on the interviews with the students about their answers in the test, it should be underlined that the answers of the students, whether it is the exams held at the school or the exams, should not remain on paper only, and that it is a necessity to scrutinize their answers. Moreover, it can be said that it will be beneficial for students to see each other's answers with the support of their teachers and discuss them constructively (without knowing whose paper it is so that they do not get offended) in terms of interpreting AE and writing VE. The fact that such environments allow students to see and correct each other's mistakes (Cobb et al., 1992) makes this suggestion meaningful. As a final emphasis, it cannot be overlooked that students who interpret and reason AE correctly and who can transfer VE to mathematics correctly will be more successful in algebra and thus in their future mathematics lives. The fact that a healthy transition from arithmetic to algebra is indispensable for future mathematics (Kieran, 2004) is one of the best grounds for this inference.

\section{Ethics Statement}

The authors confirm that the entire process of the research (including data collection) is conducted according to ethical rules. 


\section{Conflict of Interest Statement}

The authors declare no conflicts of interest.

\section{About the Authors}

Emrullah Erdem is an Associate Professor Doctor at the Department of Mathematics Education Faculty of Education at Adiyaman University in Turkey. His research is mainly on mathematical reasoning and concept definition. He has national and international articles, books, book chapters, and papers in national and international conferences.

Şeyda Zengin is a mathematics teacher at a middle school affiliated with the Ministry of National Education and a doctoral student at Adryaman University. She has articles and presentations on mathematics education.

Hayrullah Erdem is a Turkish-language teacher at a middle school affiliated with the Ministry of National Education. He also successfully graduated from the non-thesis master's program.

\section{References}

Aiken, L. R. (1971). Verbal factors and mathematics learning: A review of research. Journal for Research in Mathematics Education, 2(4), 304-313.

Akkan, Y., Baki, A., \& Çakıroğlu, Ü. (2012). Examination of the 5th-8th grade students' transition process from arithmetic to algebra with regard problem solving. Hacettepe University Journal of Education, 43, 1-13.

Bağdat, O. \& Anapa-Saban, P. (2014). Investigation of the 8th grade students' algebraic thinking skills with solo taxonomy. The Journal of Academic Social Science Studies, $26,473-496$.

Birgin, O. \& Demirören, K. (2020). Investigation of 7th and 8th grade students' performance about algebraic expressions. Pamukkale University Journal of Education, $50,99-117$.

Birgisdottir, F., Gestsdottir, S., \& Geldhof, G. J. (2020). Early predictors of first and fourth grade reading and math: The role of self-regulation and early literacy skills. Early Childhood Research Quarterly, 53, 507-519.

Booth, L. R. (1988). Children's difficulties in beginning algebra. In A. F. Coxford \& A. P. Shulte (Eds.), The ideas of algebra, K-12 (1988 yearbook) (pp. 20-32). Reston, VA: National Council of Teachers of Mathematics.

Bozkurt, A. (2018). An evaluation of the 6th grade mathematics textbook in terms of aim, student work format and applicability. Electronic Journal of Social Sciences, 17(66), 535-548.

Büyüköztürk, Ş. (2011). Data analysis handbook for social sciences, statistics- Research designSPSS applications and interpretation (14th Edition). Ankara: Pegem Academy

Cobb, P., Yackel, E., \& Wood, T. (1992). Interaction and learning in mathematics classroom situations. Educational Studies in Mathematics, 23, 99-122. 
Creswell, J. W. (2003). Research design: Qualitative, quantitative, and mixed methods approaches (2nd ed.). Thousand Oaks, CA: Sage.

Danaei, D., Jamali, H. R., Mansourian, Y., \& Rastegarpour, H. (2020). Comparing reading comprehension between children reading augmented reality and print storybooks. Computers E Education, 153, 103900.

Dikkartın-Övez, F. T. \& Çınar, B. A. (2018). Assessment of secondary school 8th grade students' algebra knowledge and algebraic thinking levels with regard to problem posing. Journal of Balikesir University Institue of Science and Technology, 20(1), 483502.

Erdem, E. (2016). Relationship between mathematical reasoning and reading comprehension: The case of the 8th grade. Necatibey Faculty of Education Electronic Journal of Science and Mathematics Education, 10(1), 393-414.

Erdem, Ö., \& Sarpkaya-Aktaş, G. (2018). Assessment of activity-based instruction in overcoming 7th grade middle school students' misconceptions in algebra. Turkish Journal of Computer and Mathematics Education, 9(2), 312-338.

Falkner, K. P., Levi, L., \& Carpenter, T. P. (1999). Children's understanding of equality: A foundation for algebra. Teaching Children Mathematics, 6(4), 232-236.

Gökkurt, B., Şahin, Ö., Soylu, Y., \& Soylu, C. (2013). Examining pre-service teachers' pedagogical content knowledge on fractions in terms of students' errors. International Online Journal of Educational Sciences, 5(3),719-735.

Han, Y., \& Ginsburg, H. P. (2001). Chinese and English mathematics language: The relation between linguistic clarity and mathematics performance. Mathematical Thinking and Learning, 3(2-3), 201-220.

Hemmi, K., Bråting, K. \& Lepik, M. (2021) Curricular approaches to algebra in Estonia, Finland and Sweden - a comparative study. Mathematical Thinking and Learning, 23(1), 49-71.

Kao, G. Y. M., Tsai, C. C., Liu, C. Y., \& Yang, C. H. (2016). The effects of high/low interactive electronic storybooks on elementary school students' reading motivation, story comprehension and chromatics concepts. Computers $\mathcal{E}$ Education, 100, 56-70.

Kieran, C. (1992). The learning and teaching of school algebra. In D.A. Grouws (Ed.), Handbook of research on mathematics teaching and learning (pp. 390-419). New York: Macmillan.

Kieran, C. (2004). Algebraic thinking in the early grades: what is it? The Mathematics Educator, 8(1), 139-151.

Kieran, C. \& Yerushalmy, M. (2004). Research on the role of technological environments in algebra learning and teaching. In K. Stacey, H. Chick and M. Kendal (Eds), The Future of the Teaching and Learning of Algebra: The 12th ICMI Study (pp. 95-152). Dordrecht, the Netherlands: Kluwer Academic.

Knopik, V. S., \& DeFries, J. C. (1999). Etiology of covariation between reading and mathematics performance: A twin study. Twin Research, 2(3), 226-234.

Leitze, A. R., \& Kitt, N. A. (2000). Using homemade algebra tiles to develop algebra and prealgebra concepts. Mathematics Teacher, 93(6), 462-466. 
Ma, X. (1995). Gender differences in mathematics achievement between Canadian and Asian education systems. The Journal of Educational Research, 89(2), 118-127.

Ministry of National Education [MNE]. (2018). Middle school mathematics 5-8. Classes teaching program. Head Council of Education and Morality.

Miles, M. B. \& Huberman, A. M. (1994). An expanded sourcebook: qualitative data analysis (2nd Editon). Thousand Oaks, CA: Sage.

Moss, J. \& Case, R. (1999). Developing children's understanding of the rational numbers: a new model and experimental curriculum. Journal for Research in Mathematics Education, 30(2), 122 - 147.

Nathan, M. J., \& Koedinger, K. R. (2000). Teachers' and researcher's beliefs about the development of algebraic reasoning. Journal for Research in Mathematics Education, 31(2), 168-190.

National Council of Teachers of Mathematics [NCTM] (2000). Principles and standards for school mathematics. Reston, VA.

Özdemir-Baki, G. \& Işık, A. (2018). Investigation of the noticing levels of teachers about students' mathematical thinking: a lesson study model. Turkish Journal of Computer and Mathematics Education, 9(1), 122-146.

Passolunghi, M. C., \& Pazzaglia, F. (2005). A comparison of updating processes in children good or poor in arithmetic word problem-solving. Learning and Individual Differences, 15(4), 257-269.

Perso, T. (1992). Using diagnostic teaching to overcome misconceptions in algebra. The Mathematical Association of Western Australia.

Rose, D. S., Parks, M., Androes, K., \& McMahon, S. D. (2000). Imagery-based learning: Improving elementary students' reading comprehension with drama techniques. The Journal of Educational Research, 94(1), 55-63.

Sharp, J. M. (1995). Results of using algebra tiles as meaningful representations of algebra concepts. Paper presented at the annual meeting of the Mid-Western Education Research Association, Chicago, IL.

Stacey, K. \& MacGregor, M. (2000). Learning the algebraic method of solving problems. Journal of Mathematical Behaviour, 18(2), 149-167.

Stephens, A. C., Knuth, E. J., Blanton, M. L., Isler, I., Gardiner, A. M., \& Marum, T. (2013). Equation structure and the meaning of the equal sign: The impact of task selection in eliciting elementary students' understandings. Journal of Mathematical Behavior, 32(2), 173-182.

Susac, A., Bubic, A., Vrbanc, A., \& Planinic, M. (2014). Development of abstract mathematical reasoning: The case of algebra. Frontiers in Human Neuroscience, 8 , 679.

Şimşek, B., \& Soylu, Y. (2018). Examination of the reasons for these mistakes made by elementary school 7 th grade students in relation to algebraic expressions. The Journal of International Social Research, 11(59), 830-848

Taylor-Cox, J. (2003). Algebra in the early years? Young Children, 58(1), 14-21. 
Tirosh, D. (2000). Enhancing prospective teachers' knowledge of children's conceptions: the case of division of fractions. Journal for Research in Mathematics Education, 31(1), $5-25$.

Ural, A., \& Ülper, H. (2013). The evaluation of the relationship between preservice elementary mathematics teachers' mathematical modeling and reading comprehension skills. Journal of Theoretical Educational Science, 6(2), 214-241.

Vilenius-Tuohimaa, P. M., Aunola, K., \& Nurmi, J. (2008). The association between mathematical word problems and reading comprehension. Educational Psychology, 28(4), 409-426.

Voutsina, C. (2019). Context variation and syntax nuances of the equal sign in elementary school mathematics. Canadian Journal of Science, Mathematics and Technology Education, 19(4), 415-429.

Yackel, E., (1997). A foundation for algebraic reasoning in the early grades. Teaching Children Mathematics, 3(6), 276-280.

Yin, R. K. (2011). Qualitative research from start to finish. New York: The Guilford Press. 
Author(s) will retain the copyright of their published articles agreeing that a Creative Commons Attribution 4.0 International License (CC BY 4.0) terms will be applied to their work. Under the terms of this license, no permission is required from the author(s) or publisher for members of the community to copy, distribute, transmit or adapt the article content, providing a proper, prominent and unambiguous attribution to the authors in a manner that makes clear that the materials are being reused under permission of a Creative Commons License. Views, opinions and conclusions expressed in this research article are views, opinions and conclusions of the author(s). Open Access Publishing Group and European Journal of Education Studies shall not be responsible or answerable for any loss, damage or liability caused in relation to/arising out of conflicts of interest, copyright violations and inappropriate or inaccurate use of any kind content related or integrated into the research work. All the published works are meeting the Open Access Publishing requirements and can be freely accessed, shared, modified, distributed and used in educational, commercial and non-commercial purposes under a Creative Commons Attribution 4.0 International License (CC BY 4.0). 